\title{
TOWARD A BUILDING OCCUPANT NETWORK AGENT-BASED MODEL TO SIMULATE PEER INDUCED ENERGY CONSERVATION BEHAVIOR
}

\author{
Jiayu Chen \\ Columbia University \\ 610 S.W. Mudd Building \\ 500 West 120th Street \\ New York, NY 10027, USA
}

\author{
John E. Taylor \\ Virginia Tech \\ 113B Patton Hall \\ Blacksburg, VA 24061, USA
}

\author{
His-Hsien Wei \\ University of Maryland \\ 1173 Glenn L. Martin Hall \\ College Park, MD 20742, USA
}

\begin{abstract}
Building occupant networks can impact energy use decision-making. With the use of eco-feedback systems, energy usage information can propagate through social networks and influence an individual's energy consumption decisions. In this paper, we develop a computational model for individual energy consumption behavior and network information transmission. By comparing the impact of several parameters in the network level computational model and validating the parameters in an experimental setting, future research can utilize this model to clarify how network relations can be leveraged for modifying energy conservation behavior.
\end{abstract}

\section{INTRODUCTION}

According to the U.S. Department of Energy's 2007 survey, nearly $40 \%$ of energy was consumed by the built environment. Nearly $21.2 \%$ of energy utilized in the U.S. was allotted to the residential sector for heating, cooling and lighting (Energy Information Administration 2009). In addition, the residential energy consumption has increased steadily in the last 20 years, especially in regard to electricity use. Nearly $38.5 \%$ of national total electricity end use was consumed by the residential sector (Energy Information Administration 2009).

At the residential building level, occupants generally have a high degree of control over their energy consumption. A growing number of scholars are studying how to reduce the energy use through solutions derived from technological and social-psychological approaches (Stern 1992; McMakin et al. 2002; Goldstein et al. 2008; Saelens et al. 2011). Some scholars have investigated patterns in energy saving behavior by providing network level information feedback systems to users (Odom et al. 2008; Khan and Canny 2008). However, more comprehensive studies on the relationship between social networks and energy use are still needed. The goal of the research presented in this paper is to develop a computational model to simulate individual energy consumption behavior to take first steps toward increasing our understanding of how network structure can affect information transmission and energy conservation behaviors. 


\section{BACKGROUND}

Energy conservation can be achieved through both modification to energy infrastructure and through modification of human behavior. For residential buildings, modifications to infrastructure include, the installation of energy efficient appliances, modern wall insulation and consumption feedback systems. On the other hand, attempts to increase energy saving through the modification to human behavior have focused on both the role that social networks (Wilson and Dowlatabadi 2007) and practice adaptation (Dalamagkidis et al. 2007; Haldi and Robinson 2008) play in influencing human behavior including the social-psychological relationship between individuals and their peers (DeMeo and Taylor 1984).

Human behaviors are strongly influenced by peers, especially when peers possess strong relationship ties, for example, peers that are neighbors, close friend or relatives. This fact has been observed by a number of scholars since the mid-1950s (e.g. Festinger 1954; Milgram et al. 1969). For instance, Göckeritz et al. (2010) proposed that the energy conservation behaviors of others have a strong positive correlation with an individual's conservation actions. Nolan et al. (2008) also showed that a normative message that contains the conservation behavior of the majority of an individual's neighbors is a strong motivator that spurs energy savings. Nolan shows that certain behaviors started by individuals spread throughout a building community and to a larger, society scale. As the behavior diffused through the peer network, social norms of conservation were established, which promoted individual conservation practices in the home.

\section{METHODOLOGY}

\subsection{Data Resource}

The simulation input data used in this paper was collected from a dormitory pilot experiment published in 2010 (Peschiera et al. 2010). The pilot study allowed us to acquire a rich set of data to construct our simulation model and to incorporate agent decision-making norms based on authentic data collected in an experimental setting. Residents' energy consumption can never be negative. Both consumers of very large and very small amounts of energy are rare, while the majority of consumers fall somewhere in between the extremes of energy use. Therefore, it is reasonable to describe energy consumption as a restricted distribution. In order to find a suitable distribution for individual energy use, we collected all usage statistics for the non-participants group, which consisted of data points for 46 days, arranged as a 2070 data point pool. After plotting the histogram and testing goodness of fit, we chose a lognormal distribution as the initial consumption generating distribution. The Kolmogorov Smirnov $p$-value is 0.03814 , which was the parameter used to evaluate goodness of fit. Given that a fitted distribution is statistically meaningful when the p-value is less than 0.05 , this result demonstrates that the chosen distribution provides both a reliable and accurate account of the energy consumption observed for the non-participant group. Figure 1 shows the distribution fitting and quantile plot, and the vertical strait line indicates the $90 \%$ and $95 \%$ confident interval. The real data show a fat tail at the extreme values, but since we only concentrate on the general situation and the tail is negatively biased, the lognormal distribution is reliable enough for the simulation. In following section, we emulate the individual's initial energy consumption based on this distribution.

\subsection{Network Energy Efficiency Model}

\subsubsection{Agent Norm}

Based on the information participants received in the pilot study, we constructed an algorithm for an individual to follow in simulation as the agent norm. First, we assumed individual's behavior will be affected by others, for example, when there has a user in their network whose use is less than the viewer, the viewer has a probability to take action to reduce electricity use. The viewers will compare their use 
with each friend and make decisions. If they find their use is already less than others, they also have a probability to increase their use or remain same. For each decision making iteration, the probability that whether they take action to save the electricity is correlated to the quality of relationship.
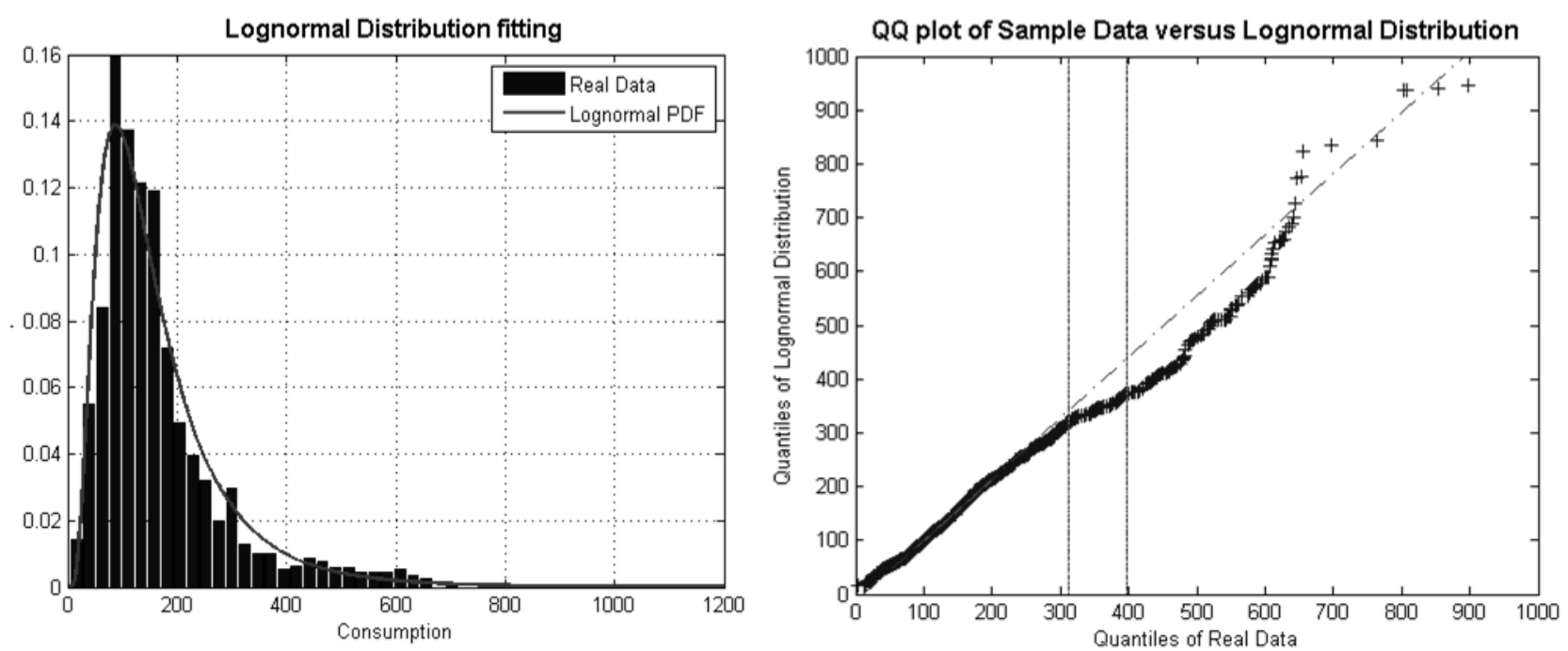

Figure 1: Energy consumption data fitting and quantile plot

\section{Algorithm (Individual decision making process and Agent Norm)}

Input: a connected social network with weight of connections and the initialized energy consumption for each agent within the network.

Output: Finalized energy consumption of each agent through a time period and the energy use record within this period.

[Initialization]

Step 1: For each agent $v_{i} \in V(G)$ where $|V(G)|=k$, have an energy consumption of $C_{i}$

Find out the neighbors of each agent and form as $N\left(v_{i}\right)$ and $v_{j}\left(v_{j} \in N\left(v_{i}\right), \forall j=1,2, \ldots n\right)$ is the neighbor of $v_{i}$ and each $v_{j}$ also have their own energy consumption $C_{j}$

Step 2: For the total simulation period

While $t<T$, record the energy consumption $E_{i t}$

If $t>=T$, go to Step 5

Step 3: For $\mathrm{j}=1: \mathrm{n}$

If $v_{i} \geq v_{j}$, generate random number $U_{1}$

If $U_{1}>\beta_{i j}, G M D_{t+1} \rightarrow C_{t+1}$

Return $E_{i t}=C_{i t}, t=t+1$, go to Step 2

Elseif $U_{1} \leq \beta_{i j}$, go to Step 4

Step 4:if $v_{i}<v_{j}$, generate random number $U_{2}$

If $U_{2}>u, C_{t} \rightarrow C_{t+1}$

Elseif $U_{2} \leq u, \quad G M I_{t+1} \rightarrow C_{t+1}$

Return $E_{i t}=C_{i t}, t=t+1$, go to Setp 2 
Step 5: Return $E_{i t}$ and Print Data

For the whole decision making process,

$$
p=\tau P\left(\beta_{n} \mid \beta_{n-1}\right) P\left(\beta_{n-1} \mid \beta_{n-2}\right) \ldots P\left(\beta_{2} \mid \beta_{1}\right)=\tau \prod_{i=1}^{n-1} P\left(\beta_{i+1} \mid \beta_{i}\right)
$$

$\mathrm{P}\left(\beta_{\mathrm{n}}\right)$ is the probability that an individual will take energy-saving action based on the consumption of an individual in their peer network to whom they have the relationship $\beta_{\mathrm{n}}$.

$n$ is the index of neighbors in the agent's network

$\tau$ is a scalar can convert edge weight to proper probability so that the model output can fit the experiment data.

For the increment and reduction of resident's energy use, we employ a Geometric Brownian Motion (GBM) process with drift. Parameters are drawn from the experiment:

$$
C_{t+1}=C_{t} \exp \left(\left[\mu-\frac{1}{2} \sigma^{2}\right]+\sigma W(T)\right)
$$

According to our model and observations from the experiment, residents may increase or decrease their consumption with respect to their network neighbors' consumption in a different pattern. However, even they saved electricity eventually, their electricity use is not necessarily monotonically increase or decrease to that results. So we simulated their consumption in two types of Geometric Brownian Motion(GBM) model: 1) The GBM that has an upward drift (GBMI) or a downward drift (GBMD) to emulate the consumption increase or decrease.

$C_{t}$ is individual's energy consumption at time t.

$W(T)$ is a normal random error with mean equal to 0 .

$\sigma$ is the standard division of energy use increment/decrement $\left(\sigma_{i}\right.$ or $\left.\sigma_{d}\right)$.

$\mu$ is the mean of energy use increment/decrement $\left(\mu_{i}\right.$ or $\left.\mu_{d}\right)$.

For GMI and GMD they will use different $\sigma$ and $\mu$ which comes from the experiment.

In above agent norm, we applied conditional probability with $\tau$ and Geometric Brownian Motion to calibrate the simulation result with experimental data (Figure 2). Since we are not certain about the direct correlation between the connection weight (relationship $\beta_{n}$ ) and individual's probability to make decisions, we try to use $\tau$ to calibrate the experimental data with simulated results. In addition, from the experimental results we know even if individuals decided to conserve the energy consumption, it does not necessarily mean they will reduce their energy monotonically. Thus, in order to catch the fluctuation of their energy consumption path, we introduced Geometric Brownian Motion in our simulation agent norm.

\subsubsection{Environment}

In this agent-based simulation, the environment in which residents act is their peer network. We assume that one agent's energy use can affect the energy use of other agents with varying degrees of probability. The quantity of consumption by neighbors of an agent's peer network may have a strong influence on modifying a given agent's energy-saving behavior. On one hand, the agent' behaviors can actively change the energy consumption patterns in the whole network, i.e. throughout the environment. On the other hand, once the environment changes, the feedback of changes will inversely affect agent's decision making processes. For example, if one agent sees its energy consumption is more than its neighbors, it may take action to reduce energy consumption. Once this change occurs, the effect of its reduced energy consumption will be incorporated into the usage statistics that are viewed by other agents in the network. This new information may provide the catalyst for other network agents to take similar energy-saving action and may trigger an "energy saving competition." 

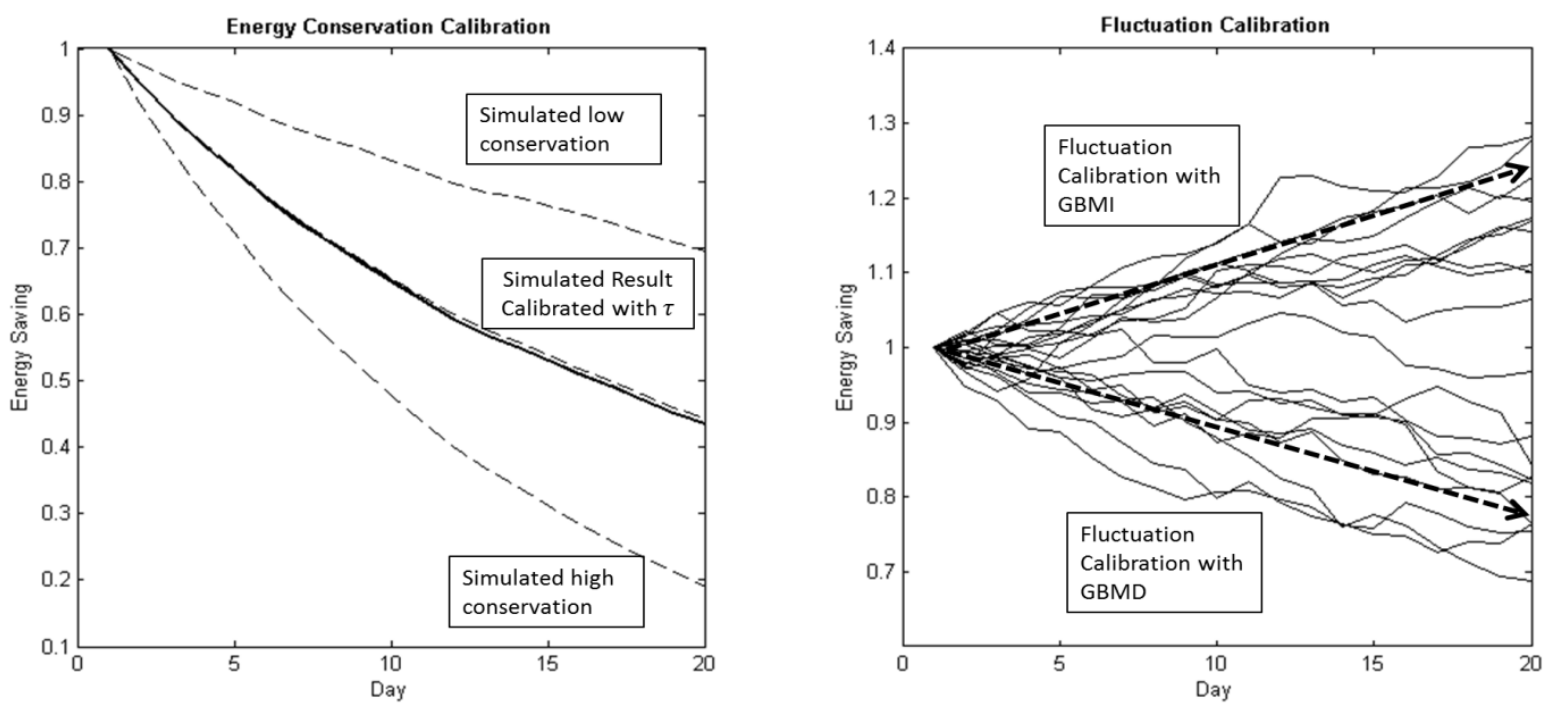

Figure 2: Simulated Result Calibration
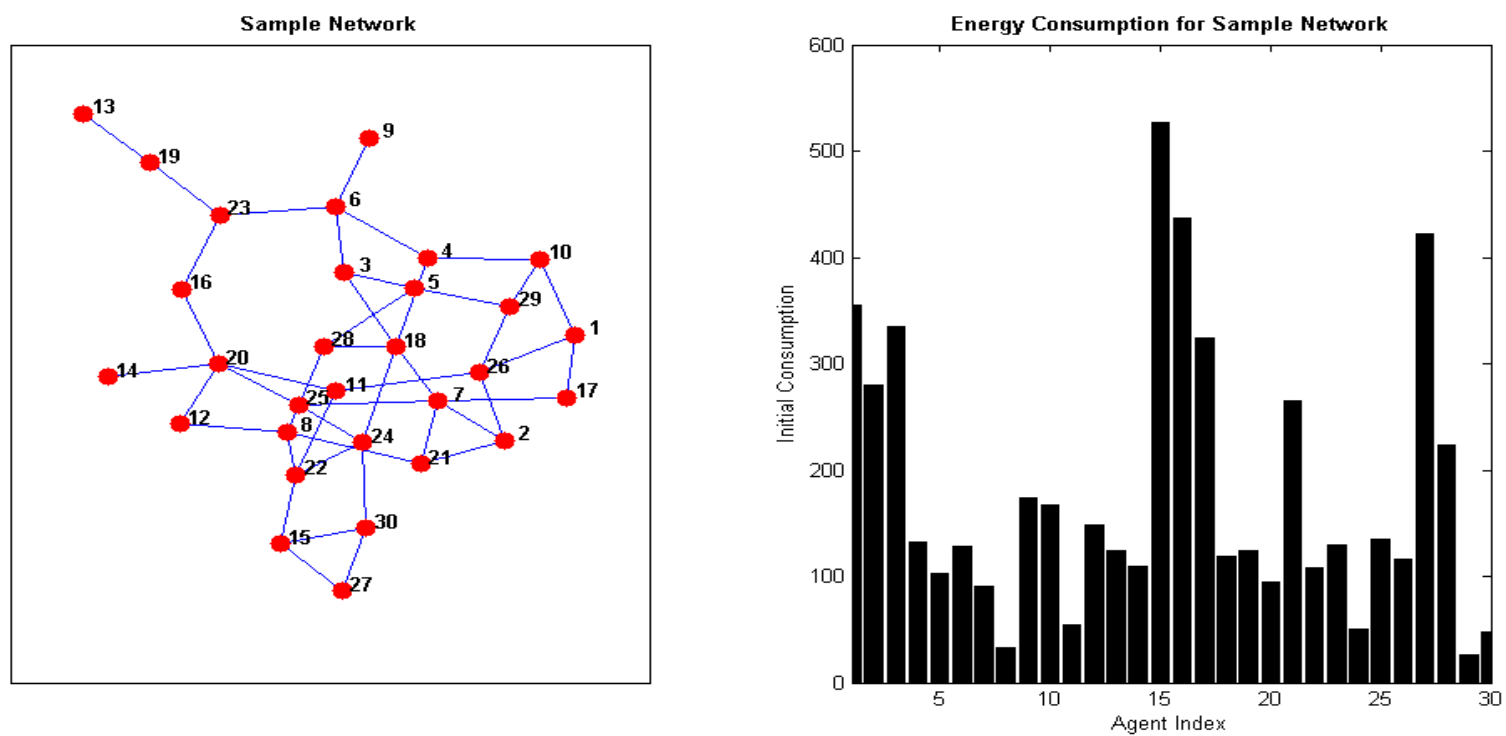

Figure 3: Sample Network Energy Consumption

In our simulation, the most important environment is the network that connected all agents. We adopt random graph theory to generate complex networks (Figure 3). Since 1950, large networks with no apparent design principles were described as random graph, which was proposed as the simplest and most straight forward realization of a complex network. According to Erdős-Rényi model, we simulated N vertex with approximately $p N(N-1) / 2$ degree, which follows Poisson distribution. In addition, recent study indicate that large network, for example, World-Wide Web, Internet, metabolic and protein networks, have the scale-free property (degree distribution has a power-law tail). However, since our residential network is relatively small compare to other networks, we only adopt random graph model without scale-free property. 


\section{SIMULATION RESULTS}

\subsection{Simulation Results}

The following Figure 4 reflects a single simulation run of all the network agents' dynamic energy consumption. Each agent can make decisions separately based on its own environment, although we assume that these decisions will affect decisions made by its neighbors in the peer network. In future research we will examine unique contributions of varying network properties on conservation behavior.

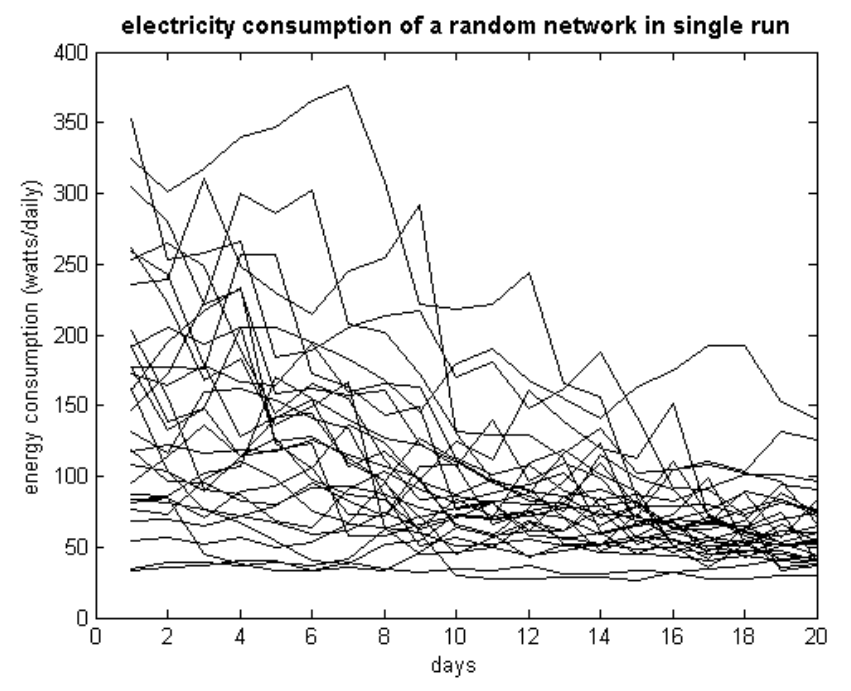

Figure 4: Energy Reduction Performance of Baseline Case

In future research we plan to employ this model of an agent's simulated energy consumption to find some meaningful difference between networks with different types of structures. We expect that network size may be one condition that would lead to increasing or decreasing energy saving behavior.

\subsection{Validation and Limitations}

The purpose of this simulation is to explore the effect of peer network structure on energy saving behavior. In order to achieve this goal, we built an agent-based decision model to estimate such behaviors using data from an energy conservation pilot study. However, this model needs further empirical validation from experimental data. Future research should also address some limitations in this research. First, we simulated the network as regular random graphs (Erdős-Rényi 1960). More recent research shows that real social networks show scaling free (Barabási and Albert 1999) and small-world (Watts and Strogatz 1998) properties. Second, we do not allow the response-relapse saving pattern to occur in our simulation, since we do not fully understand the mechanism of energy use relapse. So, to simplify the model, we assumed the agent behavior stays consistent throughout the simulated period. If we expect a more effective and long-lasting behavior of the simulation with response-relapse pattern embedded, more research is required.

\section{DISCUSSION}

The research described in this paper presents an agent-based simulation model that explores the relationship between peer networks in buildings and energy conservation behaviors of building occupants. Energy efficiency is seldom studied at the peer network level. Although experimental research recently has found that sharing energy use information through social networks promotes energy saving by building occupants (Petersen et al. 2008; Peschiera et al. 2010). However, the shape and character of 


\section{Chen, Taylor and Wei}

social networks vary from building to building, which means that the relationship between peer network structure and energy saving behavior is difficult to generalize based solely on experimental data without the aid of simulations. Thus, a model that can quantitatively explain the residents' decision making process is a critical contribution to our understanding of how best to leverage the interpersonal relationships captured by peer networks to encourage energy savings.

\section{REFERENCES}

Barabási, A. and R. Albert. 1999. "Emergence of Scaling in Random Networks" Science 286(5439): 509512

Dalamagkidis, K., D. Kolokotsa, K. Kalaitzakis and G. Stavrakakis. 2007. "Reinforcement learning for energy conservation and comfort in buildings." Building and Environment, 42(7):2686-2598.

DeMeo, E., and R. Taylor. 1984. "Solar Photovoltaic Power Systems: An Electric Utility R \& D Perspective." Science, 224(4646): 245-251.

Erdős, P. and A. Rényi 1960. "On the evolution of random graphs." Publ. Math. Inst. Hung. Acad. Sci, 5: $17-61$

Festinger, L. 1954. “A theory of social comparison processes.” Human Relations, 7: 117-140

Goldstein, N., R. Cialdini and V. Griskevicius. 2008. "A room with a viewpoint: Using social norms to motivate environmental conservation in hotels." Journal of Consumer Research, 35(3): 472-482.

Göckeritz, S., P. W. Schultz, T. Rendón, R. Cialdini, N. J. Goldstein and V. Griskevicius. 2010. "Descriptive normative beliefs and conservation behavior: the moderating roles of personal involvement and injunctive normative beliefs." European Journal of Social Psychology, 40: 514523.

Haldi, F. and D. Robinson. 2008. "On the behaviour and adaptation of office occupants." Building and Environment, 43(12):2163-2177.

Khan, O. and J. Canny. 2008. "Promoting environmentally sustainable behaviors using social marketing in emerging persuasive technologies.'In Proceedings of Sixth International Conference on Pervasive Computing, 75-78, Sydney.

McMakin, A., E. Malone and R. Lundgren. 2002. "Motivating residents to conserve energy without financial incentives." Environment and Behavior, 34(6): 848-863.

Milgram, S., L. Bickman, and L. Berkowitz 1969. "Note on the drawing power of crowds of different size." Journal of Personality and Social Psychology, 13: 79-82.

Nolan, J., P. Schultz, R. Cialdini, N. Goldstein and V. Griskevicius. 2008. "Normative social influence is underdetected." Personality and Social Psychology Bulletin, 34(7): 913-923.

Odom, W., J. Pierce and D. Roedl. 2008. "Social Incentive \& Eco-Visualization Displays: Toward Persuading Greater Change in Dormitory Communities." Workshop at OZCHI 2008. ACM press

Peschiera, G., J. E. Taylor and J. A. Siegel. 2010. "Response-relapse patterns of building occupant electricity consumption following exposure to personal, contextualized and occupant peer network utilization data." Energy and Building, 42(8):1329-1336.

Petersen, J., V. Shunturov, K. Janda, G. Platt, and K. Weinberger 2007. "Dormitory residents reduce electricity consumption when exposed to real-time visual feedback and incentives." International Journal of Sustainability in Higher Education, 8(1): 16-33.

Saelens, D., W. Parys and R. Baetens. 2011. "Energy and comfort performance of thermally activated building systems including occupant behavior." Building and Environment, 46(4):835-848.

Stern, P. 1992. "What psychology knows about energy conservation." American Psychologist 47: 12241232.

Watts, D. and S. Strogatz. 1998. "Collective dynamics of small-world networks." Nature, 393:440-442

Wilson, C. and H. Dowlatabadi. 2007. "Models of decision making and residential energy use." Annual Review of Environment and Resources, 32(1): 169-203. 


\section{AUTHOR BIOGRAPHIES}

JIAYU CHEN is a Graduate Research Assistant in the Civil Engineering and Engineering Mechanics Department at Columbia University. He conducts simulation research in the Project Network Dynamics Lab on the agent-based simulation of peer networks to enhance energy efficiency. His email is jc3252@columbia.edu.

JOHN E. TAYLOR is an Associate Professor in the Charles E. Via, Jr. Department of Civil and Environmental Engineering at Virginia Tech. He received his Ph.D. from Stanford University in 2006. $\mathrm{He}$ is Director of the Civil Engineering Network Dynamics Lab at Virginia Tech and specializes in the investigation of network dynamics of industrial and societal importance. His current research focuses on three key network dynamics; (1) achieving sustained energy conservation in the built environment by coupling energy use and energy conservation practices with building occupant networks, (2) the trending increase in global outsourcing of complex engineering services, and (3) examining the impact of integrated information systems on project networks and the associated virtualization of the engineering workforce. His email is jet@vt.edu.

HSI-HSIEN WEI is a Graduate Student in the Civil and Environmental Engineering Department at the University of Maryland. His email is hw2208@umd.edu. 